\title{
SPS 界面反应增强机制调控的软磁复合材料磁性能和电阻率
}

\author{
赵占奎，李 涛，鲁书含，王明罡，张京京，程道文，吴臣，迟 悦，王虹力 \\ (长春工业大学 材料科学与工程学院, 先进结构材料教育部重点实验室, 长春 130012)
}

摘 要: 设计软磁复合材料(SMCs)的绝缘层要兼顾软磁性能和电阻率。本研究以 $\mathrm{Fe} / \mathrm{Ni}_{0.5} \mathrm{Zn}_{0.5} \mathrm{Fe}_{2} \mathrm{O}_{4}$ 复合体系为例, 研究界面 $\mathrm{MnO}_{2}$ 氧化剂对样品软磁性能和电阻率的影响, 揭示提高软磁性能和电阻率的 SMCs 界面放电等离子烧结 (SPS) 氧化还原机制。采用球磨法制备添加 $0 、 0.1 \mathrm{wt} \% 、 0.3 \mathrm{wt} \% 、 0.5 \mathrm{wt} \%$ 和 $1.0 \mathrm{wt} \% \mathrm{MnO}_{2}$ 的核壳结构 $\mathrm{Fe} @ \mathrm{Ni}_{0.5} \mathrm{Zn}_{0.5} \mathrm{Fe}_{2} \mathrm{O}_{4}\left(\mathrm{MnO}_{2}\right)$ 复合粉末, 随后 SPS 烧结制备 $\mathrm{Fe} / \mathrm{Ni}_{0.5} \mathrm{Zn}_{0.5} \mathrm{Fe}_{2} \mathrm{O}_{4}\left(\mathrm{MnO}_{2}\right)$ 块体 SMCs 样品, 通过扫描电镜 (SEM)、X 射线衍射(XRD)表征该样品的结构特征, 用精密电阻测试仪和振动样品磁强计测试该样品的电阻率和磁 性能。研究发现, 添加 $0.5 \mathrm{wt} \% \mathrm{MnO}_{2}$ 的 $\mathrm{Fe} / \mathrm{Ni}_{0.5} \mathrm{Zn}_{0.5} \mathrm{Fe}_{2} \mathrm{O}_{4}\left(\mathrm{MnO}_{2}\right)$ 块体 $\mathrm{SMCs}$ 样品比未添加样品电阻率提高 $33.7 \%$ 、 饱和磁化强度提高 6.9\%。研究结果表明, SPS 烧结增强 SMCs 界面快速氧化还原反应, $\mathrm{MnO}_{2}$ 氧化剂的添加使界面 铁氧体离子浓度变化, 降低了 $\mathrm{B}$ 位电子跃迁频率, 提高有效波尔磁子数及 B-B 磁超交换作用, 表现出同时提高 SMCs 的软磁性能和电阻率的多重效应。

关 键 词: 软磁复合材料; 放电等离子烧结; 界面反应; 电阻率; 磁性能

中图分类号: TB33; TM271 文献标识码: A

\section{Magnetic Properties and Resistivity of Soft Magnetic Composites Regulated by SPS Enhanced Interface Reaction Mechanism}

\author{
ZHAO Zhankui, LI Tao, LU Shuhan, WANG Minggang, ZHANG Jingjing, \\ CHENG Daowen, WU Chen, CHI Yue, WANG Hongli
}

(Key Laboratory of Advanced Structural Materials, Ministry of Education, School of Materials Science and Engineering, Changchun University of Technology, Changchun 130012, China)

\begin{abstract}
The design trend of the insulating layer of soft magnetic composite materials (SMCs) is to consider both their soft magnetic properties and resistivity. In this study, using $\mathrm{Fe} / \mathrm{Ni}_{0.5} \mathrm{Zn}_{0.5} \mathrm{Fe}_{2} \mathrm{O}_{4}$ composite system as an example, the influence of $\mathrm{MnO}_{2}$ oxidant on the soft magnetic properties and resistivity of the samples is studied, and the redox mechanism resulting from spark plasma sintering (SPS) at SMCs interface which improves the soft magnetic properties and resistivity is revealed. The $\mathrm{Fe} @ \mathrm{Ni}_{0.5} \mathrm{Zn}_{0.5} \mathrm{Fe}_{2} \mathrm{O}_{4}\left(\mathrm{MnO}_{2}\right)$ core-shell composite powders with different amount of $\mathrm{MnO}_{2}(0,0.1 \mathrm{wt} \%, 0.3 \mathrm{wt} \%, 0.5 \mathrm{wt} \%, 1.0 \mathrm{wt} \%)$ are prepared by ball milling, the $\mathrm{Fe} / \mathrm{Ni}_{0.5} \mathrm{Zn}_{0.5} \mathrm{Fe}_{2} \mathrm{O}_{4}\left(\mathrm{MnO}_{2}\right)$ bulk SMCs are prepared by SPS. Their structures are characterized by SEM and XRD, and their electromagnetic properties are tested by precise resistance tester and vibrating sample magnetometer. It is found that the resistivity of $\mathrm{Fe} / \mathrm{Ni}_{0.5} \mathrm{Zn}_{0.5} \mathrm{Fe}_{2} \mathrm{O}_{4}\left(\mathrm{MnO}_{2}\right)$ bulk SMCs with $0.5 \mathrm{wt} \% \mathrm{MnO}_{2}$ is $33.7 \%$ higher than that without $\mathrm{MnO}_{2}$, the saturation magnetic induction strength is $6.9 \%$ higher. The results show that the SPS sintering enhances the rapid REDOX reaction at the SMCs interface, and the addition of $\mathrm{MnO}_{2}$ oxidants changes the ions concentration of ferrite at the
\end{abstract}

收稿日期: 2020-02-03; 收到修改稿日期：2020-03-04

基金项目: 国家自然科学基金项目(51671035, 51601018, 51608050)

National Natural Science Foundation of China(51671035, 51601018, 51608050)

作者简介: 赵占奎(1969-), 男, 教授. E-mail: zhaozk@ccut.edu.cn

ZHAO Zhankui(1969-), male, professor. E-mail: zhaozk@ccut.edu.cn 
SMCs interface, shows multiple effects of simultaneously improving the soft magnetic properties and resistivity of SMCs, because it reduces the B-bit electron transition frequency, increases the effective Bohr magneton number, and improves the B-B magnetic superexchange.

Key words: soft magnetic composite materials; spark plasma sintering; interface reaction; resistivity; magnetic properties

近年来, 随着电磁驱动和半导体升级换代要求 软磁材料具备高频、小型和高效化 ${ }^{[1]}$ 。研究和开发新 的软磁复合材料(SMCs)成为国内外研究的热点 ${ }^{[2-3]}$ 。 存在于传统 SMCs 中的非磁性粘结剂和无机介电绝 缘层恶化了材料的磁性能, 导致饱和磁化强度 $(M \mathrm{~s})$ 降低，同时也存在退磁场现象，降低磁导率、增大矫 顽力 ${ }^{[4]}$ 。软磁复合材料的绝缘层应兼具高效阻断粒 间泞流和耦合铁磁颗粒间的磁场的双重作用 ${ }^{[5]}$ 。软 磁铁氧体既有高的电阻率又有高的磁导率，是理想 的绝缘层材料 ${ }^{[6-9]}$, 但由于粘结剂及孔隙问题降低 性能。本课题组以 $\mathrm{Zn}-\mathrm{Ni}$ 铁氧体作为绝缘包覆层 ${ }^{[10]}$, 采用放电等离子烧结(SPS), 在无非磁性添加剂条 件下制备了致密度达到 $98.5 \%$ 的 $\mathrm{Fe} / \mathrm{Ni}_{0.5} \mathrm{Zn}_{0.5} \mathrm{Fe}_{2} \mathrm{O}_{4}$ 双磁性组分 SMCs 材料, 具有高的电阻率和磁导率, 解决了退磁场问题，但受 SPS 放电条件下界面原子 扩散和反应增强 ${ }^{[11]}$ 的影响, 绝缘层的电阻率还亟待 提高。在 SPS 条件下如何提高 SMCs 的电阻率尚未 见报导。

本研究在绝缘层中引入少量 $\mathrm{MnO}_{2}$ 氧化剂参与 SPS 界面反应。研究氧化剂的添加量对铁氧体离子 占位的影响, 优化界面的磁学和电学的综合性能, 为新型高性能软磁复合材料的研究与开发提供理论 依据。

\section{1 实验方法}

实验所用原料为粒度小于 $45 \mu \mathrm{m}$ 的气雾化纯铁 粉末(由无锡市赛瑞金属粉末制造有限公司生产), $\mathrm{Ni}_{0.5} \mathrm{Zn}_{0.5} \mathrm{Fe}_{2} \mathrm{O}_{4}$ 铁氧体纳米粉末(由东磁股份有限公 司提供), 分析纯 $\mathrm{MnO}_{2}$ 晶体。用不锈钢球磨罐, 在 球磨机( $\mathrm{QM}-3 \mathrm{SP} 04$, 南大仪器)上以 $400 \mathrm{r} / \mathrm{min}$ 转速, 球料比 $10: 1$, 将 $\mathrm{MnO}_{2}$ 晶体球磨 $10 \mathrm{~h}$ 至纳米级。取 出球磨后的 $\mathrm{MnO}_{2}$ 粉末放入真空干燥箱中进行干燥 处理。然后将干燥后的 $\mathrm{MnO}_{2}$ 粉末分别以 $0 、 0.1 \mathrm{wt} \%$ 、 $0.3 \mathrm{wt} \% 、 0.5 \mathrm{wt} \% 、 1.0 \mathrm{wt} \%$ 比例添加至 $\mathrm{Ni}-\mathrm{Zn}$ 铁氧体 粉末中球磨混合(球料比 $1: 1$, 转速 $150 \mathrm{r} / \mathrm{min}$ ) $1 \mathrm{~h}$ 制 备包覆剂。以纯 $\mathrm{Fe}$ 粉和不同成分包覆剂按 $10: 1$ 比 例球磨(球料比 $1: 1$, 转速 $200 \mathrm{r} / \mathrm{min}$ ) $0.5 \mathrm{~h}$, 进行绝 缘包覆。最后取每种包覆粉末各 $3 \mathrm{~g}$ 在放电等离子
烧结系统(Model SPS-625 日本新特科株式会社)上 进行烧结, 采用 $\phi 15 \mathrm{~mm}$ 硬质合金磨具, 升温速率 $100{ }^{\circ} \mathrm{C} / \mathrm{min}$, 烧结温度 $600{ }^{\circ} \mathrm{C}$, 保温 $3 \mathrm{~min}, 100 \mathrm{MPa}$ 单轴压力。

采用 SEM 和 XRD 分别表征包覆前后粉末和放 电等离子烧结后样品的微观形貌和物相结构。通过 精密直流电阻测试仪(AT512, 常州安柏精密仪器有 限公司)测量样品的电阻并计算电阻率。在管式炉中 对不同 $\mathrm{MnO}_{2}$ 添加量的 SMCs 样品 $350{ }^{\circ} \mathrm{C}$ 退火 $1 \mathrm{~h}$ ( $\mathrm{Ar}$ 气氛)。采用振动样品磁强计(7404-VSM, Lake Shore)测试样品的磁性能。

\section{2 结果与讨论}

图 1 为气雾化 $\mathrm{Fe}$ 粉和经 $\mathrm{Ni}_{0.5} \mathrm{Zn}_{0.5} \mathrm{Fe}_{2} \mathrm{O}_{4}(0.5 \mathrm{wt} \%$ $\mathrm{MnO}_{2}$ ) 包覆的 $\mathrm{Fe}$ 粉的 SEM 照片, 从图 1(a) 可以看出, 纯 $\mathrm{Fe}$ 粉表面光滑, 呈现圆球状。从图 1(b)中可以看 到通过球磨实现了以少量添加 $\mathrm{MnO}_{2}$ 粉末的 $\mathrm{Ni}-\mathrm{Zn}$ 铁氧体纳米粉为包覆剂的纯铁粉表面均匀完整的包覆。

图 2 为 SPS 烧结后 $\mathrm{Fe} / \mathrm{Ni}_{0.5} \mathrm{Zn}_{0.5} \mathrm{Fe}_{2} \mathrm{O}_{4}(0.5 \mathrm{wt} \%$ $\mathrm{MnO}_{2}$ )块体 SMCs 的 SEM 照片，从照片中可以看到 样品烧结致密, 无明显孔隙, 纯铁颗粒被绝缘包覆 层隔开，包覆层均匀完整。

图 3 为 $\mathrm{Ni}_{0.5} \mathrm{Zn}_{0.5} \mathrm{Fe}_{2} \mathrm{O}_{4}$ 包覆剂粉末、未添加和 添加 $0.5 \mathrm{wt} \% \mathrm{MnO}_{2}$ 的 $\mathrm{Ni}_{0.5} \mathrm{Zn}_{0.5} \mathrm{Fe}_{2} \mathrm{O}_{4}$ 包覆的纯 $\mathrm{Fe}$ 粉 末 SPS 烧结前后样品的 XRD 图谱。图中发现, $\mathrm{Ni}_{0.5} \mathrm{Zn}_{0.5} \mathrm{Fe}_{2} \mathrm{O}_{4}$ (未添加和添加 $0.5 \mathrm{wt} \% \mathrm{MnO}_{2}$ ) 包覆纯 $\mathrm{Fe}$ 粉末及烧结样品的尖晶石相衍射峰位与原料
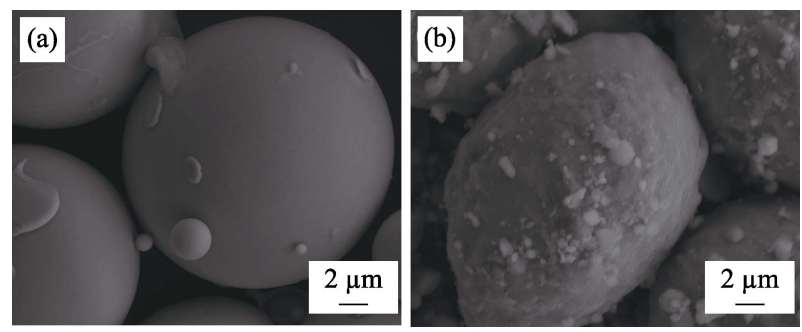

图 1 气雾化 $\mathrm{Fe}$ 粉(a)和经 $\mathrm{Ni}_{0.5} \mathrm{Zn}_{0.5} \mathrm{Fe}_{2} \mathrm{O}_{4}\left(0.5 \mathrm{wt} \% \mathrm{MnO}_{2}\right)$ 包 覆的 $\mathrm{Fe}$ 粉(b)的 SEM 照片

Fig. 1 SEM secondary electron images of air atomized $\mathrm{Fe}$ powder (a) and pure Fe powder (b) coated with $\mathrm{Ni}_{0.5} \mathrm{Zn}_{0.5} \mathrm{Fe}_{2} \mathrm{O}_{4}$ $\left(0.5 \mathrm{wt} \% \mathrm{MnO}_{2}\right)$ 


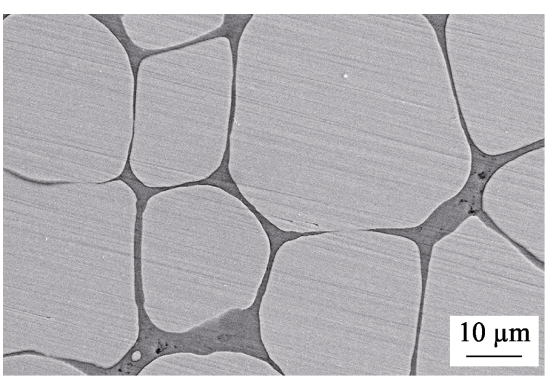

图 2 SPS 烧结 $\mathrm{Fe} / \mathrm{Ni}_{0.5} \mathrm{Zn}_{0.5} \mathrm{Fe}_{2} \mathrm{O}_{4}\left(0.5 \mathrm{wt} \% \mathrm{MnO}_{2}\right)$ 块体 $\mathrm{SMCs}$ 的 SEM 照片

Fig. 2 SEM secondary electron image of $\mathrm{Fe} / \mathrm{Ni}_{0.5} \mathrm{Zn}_{0.5} \mathrm{Fe}_{2} \mathrm{O}_{4}$ $\left(0.5 \mathrm{wt} \% \mathrm{MnO}_{2}\right)$ bulk soft magnetic composite sintered by SPS

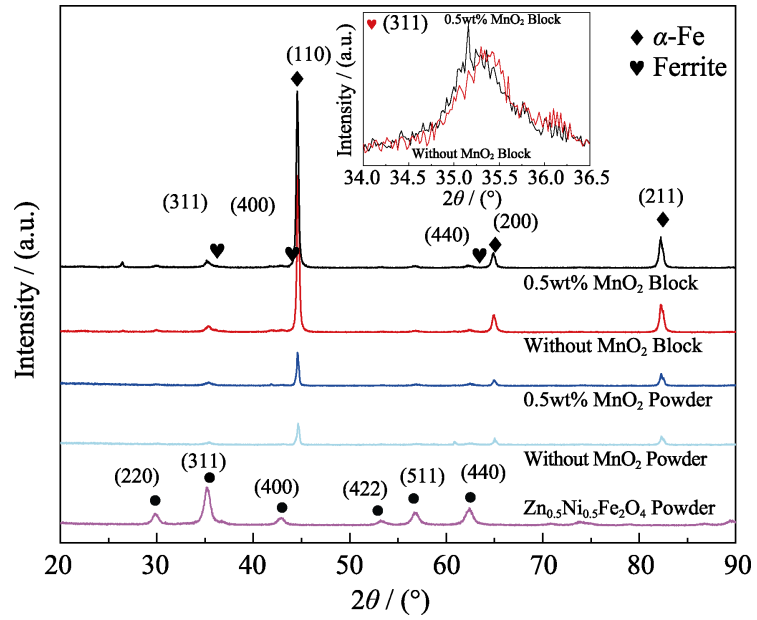

图 3 原始 $\mathrm{Ni}_{0.5} \mathrm{Zn}_{0.5} \mathrm{Fe}_{2} \mathrm{O}_{4}$ 粉末、未添加和添加 $0.5 \mathrm{wt} \% \mathrm{MnO}_{2}$ 的 $\mathrm{Ni}_{0.5} \mathrm{Zn}_{0.5} \mathrm{Fe}_{2} \mathrm{O}_{4}$ 包覆的纯 $\mathrm{Fe}$ 粉末及其 SPS 烧结后块体样 品的 XRD 图谱

Fig. 3 XRD curves of raw $\mathrm{Ni}_{0.5} \mathrm{Zn}_{0.5} \mathrm{Fe}_{2} \mathrm{O}_{4}$ powder, pure iron powder coated with $\mathrm{Ni}_{0.5} \mathrm{Zn}_{0.5} \mathrm{Fe}_{2} \mathrm{O}_{4}$ (without and with $0.5 \mathrm{wt} \%$ $\mathrm{MnO}_{2}$ ) and SPS sintered block samples

$\mathrm{Ni}_{0.5} \mathrm{Zn}_{0.5} \mathrm{Fe}_{2} \mathrm{O}_{4}$ 包覆剂粉末的衍射峰峰位一一对应, 说明包覆前后及烧结前后材料中尖晶石结构铁氧体 相没有分解。本实验加入 $\mathrm{MnO}_{2}$ 的质量占比小，几 乎观察不到衍射峰。为了探究 SPS 烧结过程中氧化 剂在绝缘界面处的行为, 仔细对比未添加和添加 $0.5 \mathrm{wt} \% \mathrm{MnO}_{2}$ 烧结后块体样品的 XRD 图谱(见图 3 插图), 发现添加 $0.5 \mathrm{wt} \% \mathrm{MnO}_{2}$ 样品比未加入样品 的尖晶石结构 $\mathrm{Ni}_{0.5} \mathrm{Zn}_{0.5} \mathrm{Fe}_{2} \mathrm{O}_{4}$ 的(311)衍射峰位发生 了左移, 说明发生了离子占位变化。这是由于 $\mathrm{MnO}_{2}$ 具有较强的氧化性, 在 SPS 场辅助作用下, 界面处 发生了氧化还原反应, 形成的 $\mathrm{Mn}^{2+}$ 半径 $(0.080 \mathrm{~nm})$ 大于 $\mathrm{Fe}^{2+}$ 半径 $(0.076 \mathrm{~nm}) 、 \mathrm{Fe}^{3+}$ 半径 $(0.064 \mathrm{~nm})$ 和 $\mathrm{Ni}^{2+}$ 半径 $(0.072 \mathrm{~nm})$, 进入铁氧体使晶格膨胀, 峰位左移。

表 1 为添加不同量 $\mathrm{MnO}_{2}$ 烧结块体样品的电阻 率和饱和磁化强度, 从表中可以看出, 电阻率随着 $\mathrm{MnO}_{2}$ 添加量的增加先升高后降低, 当添加量为 $0.5 \mathrm{wt} \%$ 时, 达到最大值 $814 \mu \Omega \cdot \mathrm{cm}$, 比相同条件未添加 $\mathrm{MnO}_{2}$ 样品的电阻率提高了 $33.7 \%$ 。
表 1 添加不同量 $\mathrm{MnO}_{2}$ 烧结块体样品 的电阻率和饱和磁化强度

Table 1 Resistivity and saturation magnetization of sintered blocks with different $\mathrm{MnO}_{2}$ content

\begin{tabular}{ccc}
\hline $\mathrm{MnO}_{2} / \mathrm{wt}^{2} \%$ & $\rho /(\mu \Omega \cdot \mathrm{cm})$ & $\mathrm{Ms} /\left(\mathrm{A} \cdot \mathrm{m} \cdot \mathrm{kg}^{-1}\right)$ \\
\hline 0 & 609 & 192.7 \\
0.1 & 687 & 199.1 \\
0.3 & 723 & 203.6 \\
0.5 & 814 & 205.9 \\
1.0 & 692 & 195.9 \\
\hline
\end{tabular}

分析认为, 在 SPS 条件下, 等离子体激活了界 面处 $\mathrm{Fe}$ 粉颗粒表面 $\mathrm{Fe}$ 原子向铁氧体界面层中扩散。 当无 $\mathrm{MnO}_{2}$ 添加时, $\mathrm{Fe}$ 原子会与尖晶石结构中的 $\mathrm{Fe}^{3+}$ 发生氧化还原反应 $\left(\mathrm{Fe}+\mathrm{Fe}^{3+} \rightarrow \mathrm{Fe}^{2+}\right)$; 当添加 $\mathrm{MnO}_{2}$ 时, $\mathrm{Mn}^{4+}$ 比 $\mathrm{Fe}^{3+}$ 的氧化性强, 促使扩散进入铁 氧体层的 $\mathrm{Fe}$ 原子优先与其反应 $\left(\mathrm{Fe}+\mathrm{Mn}^{4+} \rightarrow \mathrm{Fe}^{3+}+\right.$ $\mathrm{Mn}^{2+}$ ), 导致界面层中离子浓度发生变化。影响尖晶 石结构铁氧体中金属阳离子占居氧原子四面体间隙 (A 位)或者八面体间隙(B 位)位置的因素有离子半 径、电子组态、静电能、极化效应和离子价键平衡 等 ${ }^{[12]}$ 。一般情况下金属离子倾向于占 $\mathrm{B}$ 位的优先顺 序为 $\mathrm{Ni}^{2+} 、 \mathrm{Mn}^{3+} 、 \mathrm{Fe}^{2+} 、 \mathrm{Fe}^{3+} 、 \mathrm{Mn}^{2+} 、 \mathrm{Zn}^{2+}$, 占 $\mathrm{A}$ 位 的优先顺序则相反。因此, 无 $\mathrm{MnO}_{2}$ 添加时 SPS 过 程中产生的 $\mathrm{Fe}^{2+}$ 优先于 $\mathrm{Fe}^{3+}$ 占据尖晶石晶胞的 $\mathrm{B}$ 位。Nahonori ${ }^{[13]}$ 的研究表明, $\mathrm{B}$ 位的 $\mathrm{Fe}^{2+}$ 和 $\mathrm{Fe}^{3+}$ 间电 子跃迁激活能很小, $\mathrm{Fe}^{2+}$ 浓度的增加会明显提高电 子跃迁频率, 降低铁氧体的电阻率。因此, 在 SPS 致密化过程中无添加 $\mathrm{MnO}_{2}$ 铁氧体绝缘层的电阻率 明显下降。当添加 $\mathrm{MnO}_{2}$ 时, $\mathrm{MnO}_{2}$ 的强氧化性减少 了 $\mathrm{Fe}^{2+}$ 的产生, 在 SPS 过程中界面扩散的 $\mathrm{Fe}$ 原子直 接被氧化为 $\mathrm{Fe}^{3+}$, 反应产物 $\mathrm{Mn}^{2+}$ 优先于 $\mathrm{Fe}^{3+}$ 占据铁 氧体晶胞的 $\mathrm{A}$ 位, $\mathrm{Fe}^{3+}$ 更多占据 $\mathrm{B}$ 位, 通过降低 $\mathrm{B}$ 位 $\mathrm{Fe}^{2+}$ 浓度提高 $\mathrm{Fe}^{3+}$ 浓度, 降低了 $\mathrm{B}$ 位 $\mathrm{Fe}^{2+}$ 和 $\mathrm{Fe}^{3+}$ 间的电子跃迁频率，进而增大界面层的电阻率。

随着 $\mathrm{MnO}_{2}$ 添加量的增加电阻率随之升高; 当 添加量超过氧化还原当量, 将使过量的 $\mathrm{Mn}^{4+}$ 固溶于 铁氧体, 根据电中性原则, $\mathrm{Mn}^{4+}$ 也可能与 $\mathrm{Ni}^{2+}$ 形成 离子对进入 $\mathrm{B}$ 位, 提高 $\mathrm{B}$ 位电子跃迁几率, 使电阻 率下降。

图 4 为样品的 VSM 测试结果, 从图中可见随着 $\mathrm{MnO}_{2}$ 添加量的增加, 样品的 $M \mathrm{~s}$ 逐渐增大。图 4 中 插图为不同 $\mathrm{MnO}_{2}$ 添加量样品的 $M \mathrm{~s}$ (数据见表 1)。

当 $\mathrm{MnO}_{2}$ 添加量为 $0.5 \mathrm{wt} \%$ 时, $M \mathrm{~s}$ 达到最大值 $205.9 \mathrm{~A} \cdot \mathrm{m}^{2} / \mathrm{kg}$, 比未添加样品提高了 $6.9 \%$ 。当添加 量增加到 $1.0 \mathrm{wt} \%$ 时, $M \mathrm{~s}$ 下降。根据曲线上升段斜率 可以发现, 随着 $\mathrm{MnO}_{2}$ 添加量的增加, 样品的磁导 


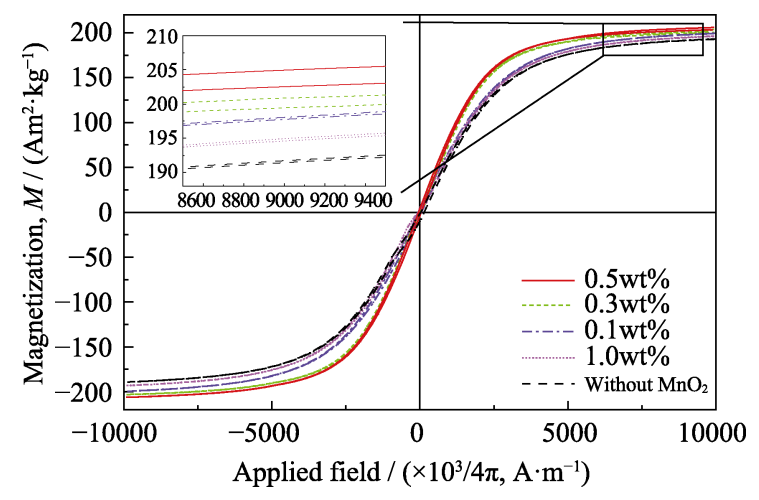

图 4 添加不同量 $\mathrm{MnO}_{2}$ 的 $\mathrm{Fe} / \mathrm{Ni}_{0.5} \mathrm{Zn}_{0.5} \mathrm{Fe}_{2} \mathrm{O}_{4}$ 经 $\mathrm{SPS}$ 烧结后 块体样品的 VSM 曲线

Fig. 4 VSM curve of SPSed $\mathrm{Fe} / \mathrm{Ni}_{0.5} \mathrm{Zn}_{0.5} \mathrm{Fe}_{2} \mathrm{O}_{4}\left(\mathrm{MnO}_{2}\right)$ blocks with different $\mathrm{MnO}_{2}$ contents

率增大, 软磁性能在多个参数上得到同时优化。分 析认为, 随着 $\mathrm{MnO}_{2}$ 的添加逐步改变了铁氧体中金 属离子的种类及占位, 对铁氧体层的磁性能具有明 显影响, 进而影响整个复合材料样品的软磁性能。

在 $\mathrm{Zn}-\mathrm{Ni}$ 铁氧体包覆层中加入了不同含量的 $\mathrm{MnO}_{2}$, 发生氧化还原反应使离子占位发生改变, 产 物中 $\mathrm{Mn}^{2+}$ 和 $\mathrm{Fe}^{3+}$ 具有 5 个波尔磁子数, $\mathrm{Mn}^{2+}$ 优先占 据 $\mathrm{A}$ 位而使更多的 $\mathrm{Fe}^{3+}$ 进入 $\mathrm{B}$ 位 ${ }^{[12]}$, 使 $\mathrm{B}$ 位上的波 尔磁子数增加, 增强了 B-B 磁超交换作用, 进而使 铁氧体绝缘层的 $M \mathrm{~s}$ 和磁导率增大, 降低了退磁场 的消极影响, 增加了铁磁颗粒之间的磁耦合效应, 矫顽力减小。当 $\mathrm{MnO}_{2}$ 添加量进一步增加, 过量的 $\mathrm{Mn}^{4+}$ 与 $\mathrm{Ni}^{2+}$ 形成离子对取代 $\mathrm{Fe}^{3+}$ 进入 $\mathrm{B}$ 位, 该离子 占位变化使有效波尔磁子数由 5 降低到 2.5 , 使 $M \mathrm{~s}$ 和磁导率减小, 矫顽力增大。因此, 加入 $\mathrm{MnO}_{2}$ 氧化 剂, 通过 SPS 烧结增强的界面反应, 改变了界面氧 化还原反应优先顺序, 改变了离子种类、浓度及占 位顺序, 具有同时提高 SMCs 电阻率和软磁性能的 多重效应。

\section{3 结论}

1)采用 SPS 烧结制备了添加 $\mathrm{MnO}_{2}$ 的 $\mathrm{Fe} / \mathrm{Ni}_{0.5} \mathrm{Zn}_{0.5} \mathrm{Fe}_{2} \mathrm{O}_{4}$ 块体 $\mathrm{SMCs}$ 。当添加 $0.5 \mathrm{wt} \% \mathrm{MnO}_{2}$ 时 SMCs 综合性能最好, 电阻率提高 $33.7 \%$, 饱和磁 化强度提高 $6.9 \%$ 。少量 $\mathrm{MnO}_{2}$ 氧化剂促使界面发
生氧化还原反应，同时提高样品的磁性能和电阻率。

2)由于 $\mathrm{MnO}_{2}$ 的强氧化性, 使界面绝缘层铁氧 体 $\mathrm{B}$ 位 $\mathrm{Fe}^{2+}$ 浓度降低, $\mathrm{Fe}^{3+}$ 浓度升高, 降低了 $\mathrm{B}$ 位离 子间电子跃迁频率，增大了界面铁氧体层的电阻率。

3)添加 $\mathrm{MnO}_{2}$ 使界面铁氧体 $\mathrm{B}$ 位有效波尔磁子 数增加, 增强了 B-B 超磁交换作用, 使铁氧体绝缘 层的饱和磁化强度和磁导率增大，增加了铁磁颗粒 之间的磁耦合效应，矫顽力减小。

\section{参考文献:}

[1] DU YOU-WEI. Progress of magnetic materials. Journal of Functional Materials, 2014(10): 1-4.

[2] SILVEYRA J M, FERRARA E, HUBER D L, et al. Soft magnetic materials for a sustainable and electrified world. Science, 2018. 362(6414): eaao0195.

[3] WU SHEN, YAN MI. Research progress on soft magnetic composites. Materials China, 2018, 37(08): 582-589.

[4] SHOKROLLAHI H, JANGHORBAN K. Soft magnetic composite materials (SMCs). Journal of Materials Processing Technology, 2007, 189(1/2/3): 1-12.

[5] FLOCH L M, MATTEI J L, LAURENT P, et al. A physical model for heterogeneous magnetic materials. Journal of Magnetism and Magnetic Materials, 1995,140-144: 2191-2192.

[6] MAEDA T, TOYODE H, IGARASHI N, et al. Development of super low iron-loss P/M soft magnetic material. SEI Technica Review, 2005, 60: 3 .

[7] LI XIAO-LONG, DONG YA-QIANG, LIU MIN, et al. New Fe-based amorphous soft magnetic composites with significant enhancement of magnetic properties by compositing with nano-(NiZn) $\mathrm{Fe}_{2} \mathrm{O}_{4}$. Journal of Journal of Alloys and Compounds, 2017, 696: 1323-1328.

[8] LI JING-XIN, YU JING, LI WANG-CHANG, et al. The preparation and magnetic performance of the iron-based soft magnetic composites with the $\mathrm{Fe} @ \mathrm{Fe}_{3} \mathrm{O}_{4}$ powder of in situ surface oxidation. Journal of Magnetism \& Magnetic Materials, 2018, 454: 103-109.

[9] LI SHI-GENG, LIU RU-TIE, XIANG XIONG. Fe-based soft magnetic composites with high permeability and low core loss by in situ coating $\mathrm{ZnFe}_{2} \mathrm{O}_{4}$ layer. Journal of Materials Science, 2020, 55(1): 274-282.

[10] WANG MING-GANG, ZHAN ZHAO, DENG NA, et al. Preparation of pure iron/Ni-Zn ferrite high strength soft magnetic composite by spark plasma sintering. Journal of Magnetism and Magnetic Materials, 2014, 361: 166-169.

[11] GAO L, MIYAMOTO H. Spark plasma sintering technology. Journal of Inorganic Materials, 1997, 12(2): 129-133.

[12] 李荫远, 李国栋. 铁氧体物理学. 北京: 科学出版社, 1978: 26-61.

[13] NAHONORI M. Electric conduction of ferrites containing $\mathrm{Fe}^{2+}$-ions. Journal of the Physical Society of Japan, 1961, 16(2): 206-208. 